\title{
Optimization methods for determination of moisture diffusivity of building materials in the drying phase
}

\author{
J. Kočí, J. Maděra, M. Jerman \& R. Černý \\ Department of Materials Engineering and Chemistry, \\ Faculty of Civil Engineering, Czech Technical University in Prague, \\ Czech Republic
}

\begin{abstract}
The contemporary laboratory experiments aimed at identification of moisture diffusivity are often conducted for the adsorption phase only. Experimental results of such designed experiments are quite simple to be analyzed by inverse analysis. However, the liquid moisture transport may exhibit significant hysteresis. Therefore the laboratory experiments should cover both adsorption (wetting) and desorption (drying) phase. In this paper we present genetic algorithm as a possible method of solution of the inverse problem of moisture transport in desorption phase. Its application is demonstrated for AAC (Autoclaved Aerated Concrete) as a one of typical building materials for ecological buildings.

Keywords: autoclaved aerated concrete, desorption, genetic algorithm, inverse analysis.
\end{abstract}

\section{Introduction}

Proper description of moisture transport within building materials is based on precise knowledge of material parameters. As far as liquid moisture transport is concerned, the key role is played by moisture diffusivity. In general, there are two types of methods for determination of moisture diffusivity under laboratory conditions - stationary and non-stationary methods. Stationary methods are unsuitable for most building materials due to their time consumption. Fortunately, there are several non-stationary methods for determination of moisture diffusivity based on principles of inverse analysis that are suitable for 
building materials. This means that for determination of moisture diffusivity it is necessary to obtain at least one moisture profile (distribution of moisture across the material).

Moisture diffusivity $\kappa$ is a material parameter characterizing the transport of liquid moisture in porous matter. One-dimensional diffusion equation describing the transport of liquid moisture can be written as

$$
\begin{gathered}
\frac{\partial u}{\partial t}=\frac{\partial}{\partial x}\left(\kappa(u) \frac{\partial u}{\partial x}\right), \\
u=\frac{m_{w}-m_{d}}{m_{d}},
\end{gathered}
$$

where $m_{w}[\mathrm{~kg}]$ is the mass of wet sample and $m_{d}[\mathrm{~kg}]$ the mass of dry sample.

The moisture diffusivity is markedly dependent on moisture content within the material. It can differ up to few orders of magnitude between dry and wet state of the same material. This fact complicates the solution of inverse problem of moisture transport and from mathematical point of view it is so-called "illposed" problem. The particular solution is usually done by simplification of initial and boundary conditions, which guarantees monotonous moisture profiles functioning as input parameters for the solution. Usually this is reached by choosing Dirichlet's boundary condition and constant initial condition. The principle of the experiment consists in obtaining moisture profiles (moisture as a function of time and position) for example by using capacitance method [1]. On such profiles the inverse analysis is applied. At these days there exist few inverse methods that can be used for determination of moisture diffusivity (e.g. Matano's method [2], double integration method [3] etc.). But as it was described above, these methods are restricted to use only for water absorption phase.

Unfortunately the above mentioned methods are possible to be used only for absorption, where the front face of the specimen is in direct contact with water (Dirichlet's boundary condition) and on the beginning of the experiment is the specimen dried out (constant initial condition). The desorption experiment is different. Although the constant initial condition could be achieved by full saturation of the specimen, the effect of Dirichlet's boundary condition is unrealistic because the material is faced to air. This implies the use of Newton's boundary condition instead; the water transfer from solid to gaseous environment is realized in the form of convective transfer of water evaporated from the surface. Therefore, for desorption phase it is impossible to use common methods as for absorption phase. As it was shown in different papers, e.g. [4], moisture diffusivity exhibits considerable effect of hysteresis, which can rise up to about one order of magnitude. Thus, it is very important to find new methods for determination of desorption curve of moisture diffusivity.

So far we used inverse analysis based on simple trial and error method, which was time consuming and also the achieved accuracy was not quite perfect [5]. Therefore we should find another solution that is more general and can be applied to a wide range of laboratory experiments. 
Among possible solutions, the genetic algorithms have big potential for solving inverse problems of moisture transport, fortunately not only for absorption phase, but for desorption phase either. In this paper we present combination of experimental and computational approach. In the experimental part laboratory experiment aimed at desorption of AAC is conducted. In the computational phase the results of laboratory experiment are investigated by inverse analysis using computer code HEMOT, which is implemented into genetic algorithm GRADE.

\section{Laboratory experiment}

In order to find moisture diffusivity of AAC a simple laboratory experiment was conducted. The experiment was aimed at moisture desorption in the material. In the experiment the rod shaped specimen was fully saturated at the beginning and it was water and waterproof insulated on four lateral sides and back side. The front side of the specimen was not insulated, thus the moisture was able to evaporate to the environment. The exchange of the moisture between the material and surrounding air was accelerated by a fan that was placed in front of the specimen (see Fig. 1).

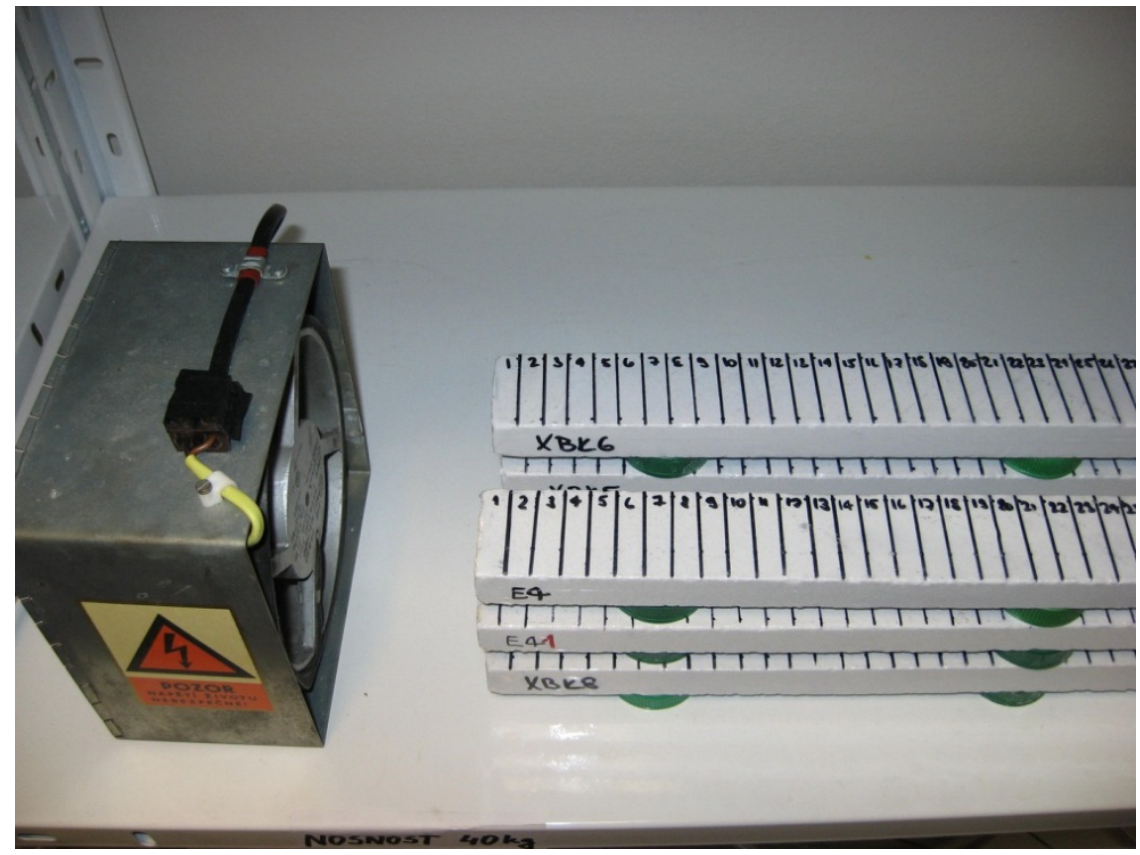

Figure 1: Scheme of the laboratory experiment.

The moisture profiles across the specimen in different time intervals were obtained using the capacitance device [1]. A relation between moisture content 
and capacitance can be determined by a calibration. The capacitance moisture meter is equipped with electrodes in the form of parallel plates with the dimensions of $20 \times 40 \mathrm{~mm}$. Because the front face of the material is in direct contact with the air, the Newton's boundary condition has to be implied. Therefore, the second objective is to determine moisture transfer coefficient for flowing air.

In the determination of moisture profiles, scanning by the capacitance moisture meter along the specimen was done in $10 \mathrm{~mm}$ steps, so that certain space averaging was done (the width of the probe is $20 \mathrm{~mm}$ ). The time intervals were chosen to cover the moisture desorption process by a sufficient number of curves.

Calibration curve of the capacitance moisture meter is for a particular material usually determined in advance using the gravimetric method. In this case, we have chosen another method. The calibration curve was determined for water suction experiment on the same material with same dimensions. The calibration was done after the moisture meter scan when the moisture penetration front was at about one half of the length of the specimen, using this scan and the results of the standard gravimetric method measurements after cutting the specimen into $10 \mathrm{~mm}$ wide pieces. The calibration curve was constructed as the dependence of the moisture content on the moisture meter reading using the data for 3 samples (see Fig. 2).

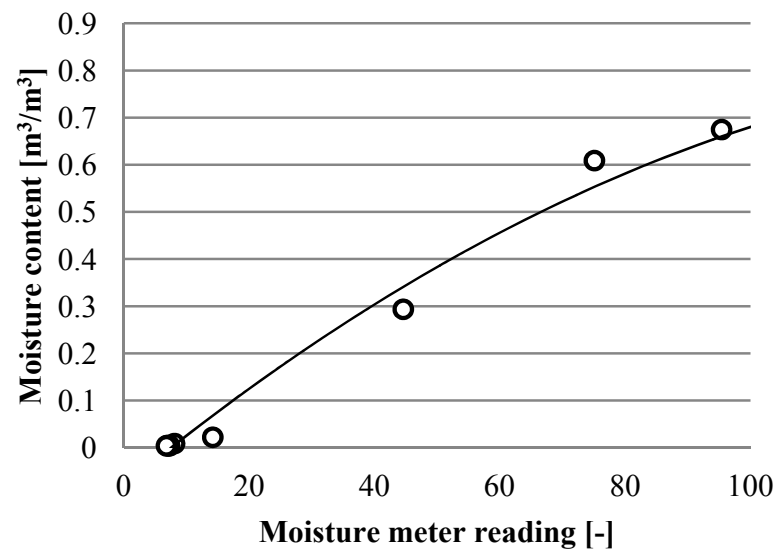

Figure 2: Calibration curve for moisture determination of AAC using the capacitance method.

During the experiment the interior conditions were kept constant: $24 \pm 1^{\circ} \mathrm{C}$ and $40 \pm 5 \%$ of relative humidity.

Results of the experiment (after calibration) are presented in Fig. 3. Although the experiment took more than 150 days, only several moisture distribution curves were chosen for the inverse analysis. The curve for 26 th day was used as initial conditions in the specimen, the other curves for 36,67 , and 89 days were fitted using genetic algorithm. 


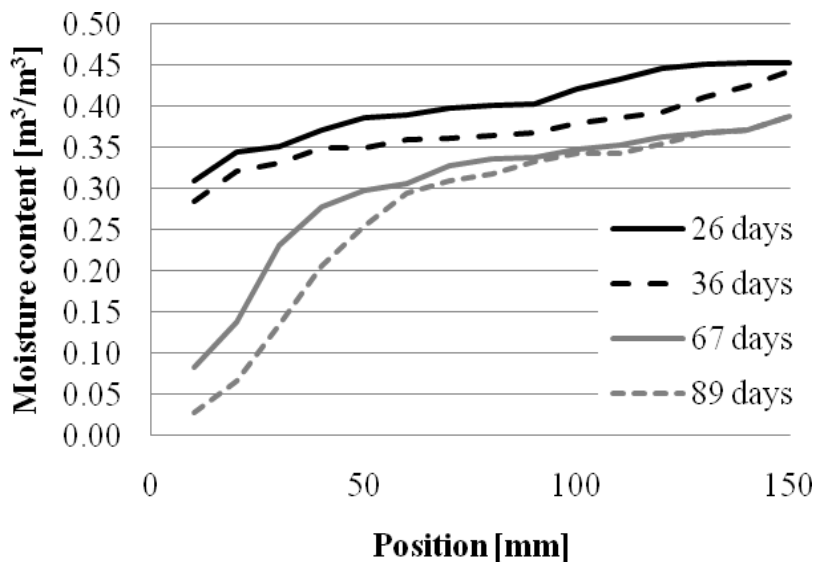

Figure 3: $\quad$ Moisture profiles in AAC during the drying experiment.

\section{Material parameters}

Basic parameters of AAC (P4-500, produced by Xella) involved in computer simulations are presented in Table 1 , where $\rho$ is the bulk density, $\psi$ the porosity, $\mu$ the water vapor diffusion resistance factor, $w_{\text {hyg }}$ the hygroscopic moisture content by volume, $c$ the specific heat capacity, $\lambda_{\text {dry }}$ the thermal conductivity in dry conditions, $\lambda_{\text {sat }}$ the thermal conductivity in water saturated conditions. These parameters were measured at Department of Materials Engineering and Chemistry, Faculty of Civil Engineering, Czech Technical University in Prague and were published in [6].

Table 1: $\quad$ Basic material parameters of AAC.

\begin{tabular}{|l|c|}
\hline$\rho\left[\mathrm{kg} / \mathrm{m}^{3}\right]$ & 500 \\
\hline$\psi[\%]$ & 80.2 \\
\hline$c[\mathrm{~J} / \mathrm{kgK}]$ & 1050 \\
\hline$\mu_{\text {dry cup }}[-]$ & 9.7 \\
\hline$\mu_{\text {wet cup }}[-]$ & 3.1 \\
\hline$\lambda_{\text {dry }}[\mathrm{W} / \mathrm{mK}]$ & 0.1138 \\
\hline$\lambda_{\text {sat }}[\mathrm{W} / \mathrm{mK}]$ & 0.7975 \\
\hline$w_{\text {hyg }}\left[\mathrm{m}^{3} / \mathrm{m}^{3}\right]$ & 0.0184 \\
\hline
\end{tabular}

\section{Computer simulation and optimization tools}

The numerical simulation tool HEMOT has been developed at the Department of Materials Engineering and Chemistry, Faculty of Civil Engineering, Czech Technical University in Prague in order to support modelling of coupled heat and water transport in porous building materials. It allows simulation of transport phenomena in constructive building details for 1D and 2D problems, whereas the 
basic variables characterizing the hygrothermal state of building constructions (temperature, moisture content, relative humidity) can be obtained as functions of space and time. The mathematical formulation of coupled heat and moisture transport equations is done according to Künzel [7] and the code works on the basis of finite element method. A particular advantage of HEMOT code is the possibility of investigation of variants concerning different constructions, different materials and different climatic loads. Constructive details of buildings and building materials can be optimized using the numerical simulation, and the reliability of constructions for different given indoor and outdoor climates can be judged. HEMOT uses the material database as a data source, which simplifies computations and allows obtaining more complex results.

For optimization of obtained results, genetic algorithm was used. Genetic algorithms belong to a group of evolution algorithms, which includes also evolution strategies and genetic programming. At present, genetic algorithms belong to the most modern optimization methods available. They follow an analogy of processes that occur in living nature within the evolution of live organisms during period of many millions of years. The principles of genetic algorithms were first proposed by Holland [8] and the details on this technique can be found in the books by Goldberg [9] and Michalewicz [10].

In genetic algorithm, a population of individuals (chromosomes), which encode candidate solutions to an optimization problem, evolves toward better solutions. The evolution usually starts from randomly generated population and happens in generations. In each generation, the fitness function of every individual in the population is evaluated, multiple individuals stochastically selected from the current population (based on their fitness) and modified using genetic operators (cross-over, mutation) to form a new population. The new population is then used in the next iteration of the algorithm. Commonly, the algorithm terminates when either a maximum number of generations has been produced (a satisfactory solution probably have not been reached), or a satisfactory fitness level has been reached for the population.

The GRADE algorithm [11] used for optimization of liquid moisture transport parameters was developed at Department of Mechanics, Faculty of Civil Engineering, Czech Technical University in Prague from its previous version called SADE [12]. This algorithm uses the simplified differential operator, but contrary to the differential evolution, the GRADE method uses the algorithmic scheme very similar to the standard genetic algorithm.

1. As the first step, the initial population is generated randomly and the objective function value is assigned to all chromosomes in the population. The size of the population is defined as the number of variables of objective function multiplied by parameter pop_rate.

2. Several new chromosomes are created using the mutation operator. Total number of mutations depends on the value of parameter called radioactivity.

3. Other new chromosomes are created using the simplified differential operator crossing-over. The total amount of chromosomes is now two times higher than in the beginning. 
4. Each newly created chromosome is evaluated by objective function.

5. The amount of chromosomes in the population is reduced by operator called selection. Hence, the amount of individuals is decreased to its original value.

Steps 2-5 are repeated until the stopping criterion is reached.

In the computational optimization by GRADE algorithm, the moisture diffusivity at desorption as a function of moisture content given by six points (reasonable compromise between the accuracy and overall time of calculation) and moisture transfer coefficient as a single value were fitted at the same time. The principles of optimization using genetic algorithm GRADE can be summarized in following few steps: In the first step, the GRADE algorithm creates an initial population of chromosomes. Each chromosome consists of seven variables of objective function - six of them represent isolated points defining moisture diffusivity function and the seventh variable represents a constant value of moisture transfer coefficient. As the pop_rate parameter is equal to 10 , the population consists of 70 chromosomes. After that, each chromosome is implemented into input file for simulation tool HEMOT. Seventy

\section{GRADE}

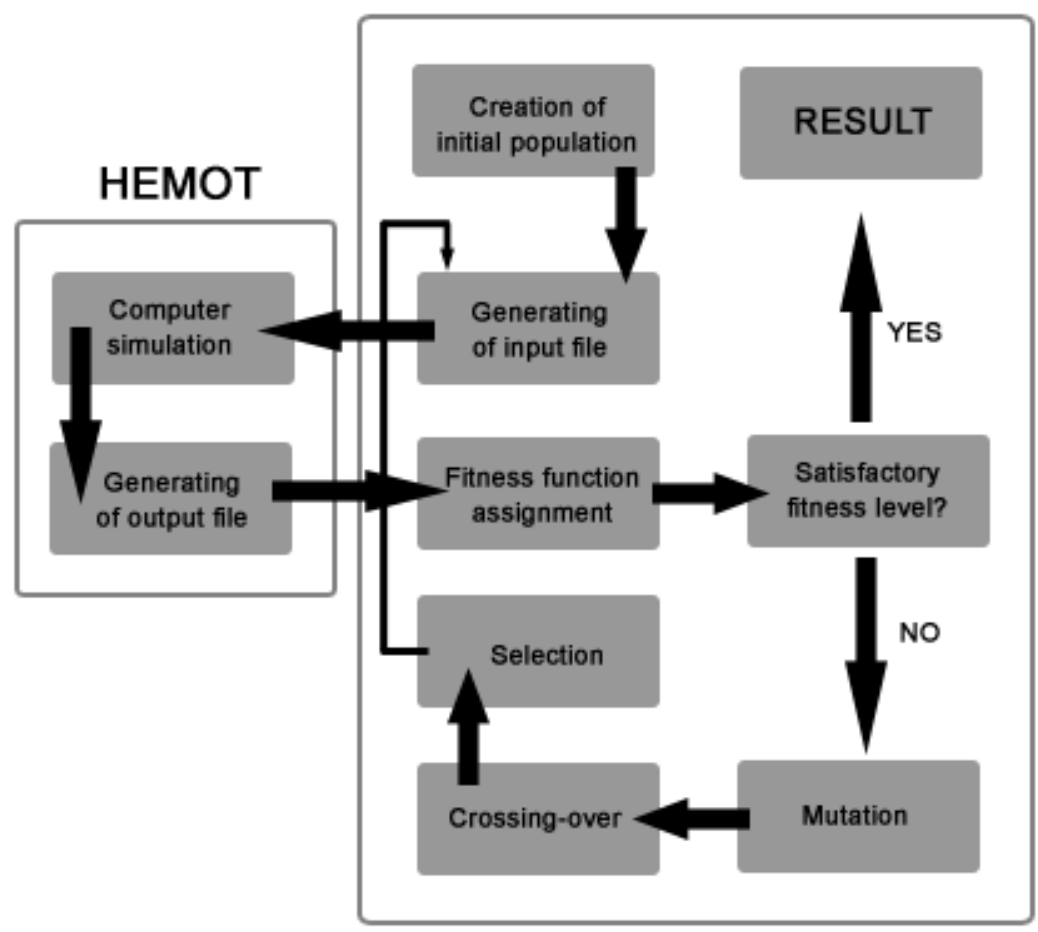

Figure 4: Scheme of optimization using GRADE algorithm and Hemot Simulation tool. 
different input files are generated in total and seventy numerical simulations are performed. Comparing the simulations results with results of laboratory experiment, the objective function can be assigned to each chromosome. The objective function was defined by principles of the least square method. In total we compared 15 points for each moisture distribution curve, this means 45 points in total (three curves). The satisfactory fitness level was set to $0.027\left(\mathrm{~m}^{3} / \mathrm{m}^{3}\right)^{2}$, which presents an average error $\pm 0.024 \mathrm{~m}^{3} / \mathrm{m}^{3}$ for each outputting value. If there is any chromosome with that satisfactory fitness level (equal or lower than 0.027), the optimization is stopped. Failing that, the genetic operators are applied and new generation of chromosomes is created. Whole process is then repeated until the stopping criterion is reached. The scheme of the optimization process is shown in Fig. 4.

\section{Optimization results and discussion}

The combined experimental/computational technique described above was used for the determination of moisture diffusivity of cellular concrete (P4-500 by Xella) at desorption (drying) as a function of moisture content. The optimization results are shown in Fig. 5, where one can see a reasonably good match between measured and optimized data.

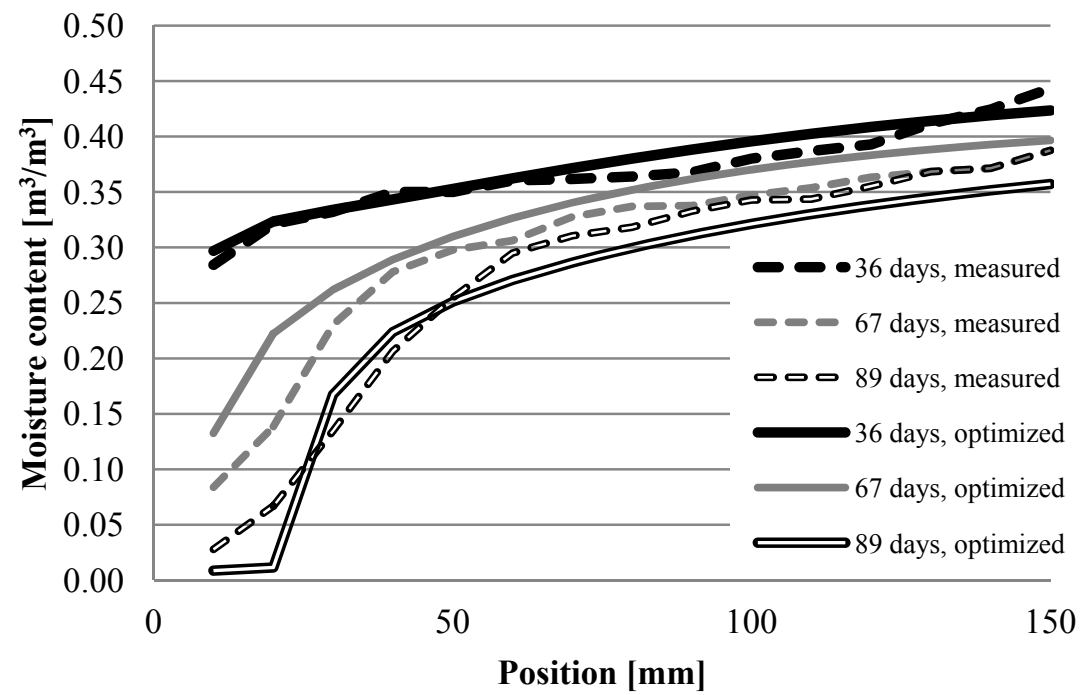

Figure 5: Optimization results of GRADE algorithm.

The optimized moisture diffusivity is shown in Fig. 6 as an average value of all found moisture diffusivity functions with satisfactory fitness level.

The optimized value of moisture transfer coefficient was found as $6.6019 \cdot 10^{-7} \mathrm{~s} / \mathrm{m}$, which is logically higher than standard value of this coefficient 
used for the interior. So far we have involved the flowing air with one velocity only. Next, we should involve also air flow with different velocities in order to determine different values of moisture transfer coefficient based on air flow characteristics. With the knowledge of moisture diffusivity in desorption phase it should be quite simple to find other values of moisture transfer coefficient, because as far as we investigate P4-500 AAC, the moisture diffusivity function stays unchanged. Therefore, the optimization process is very simplified.

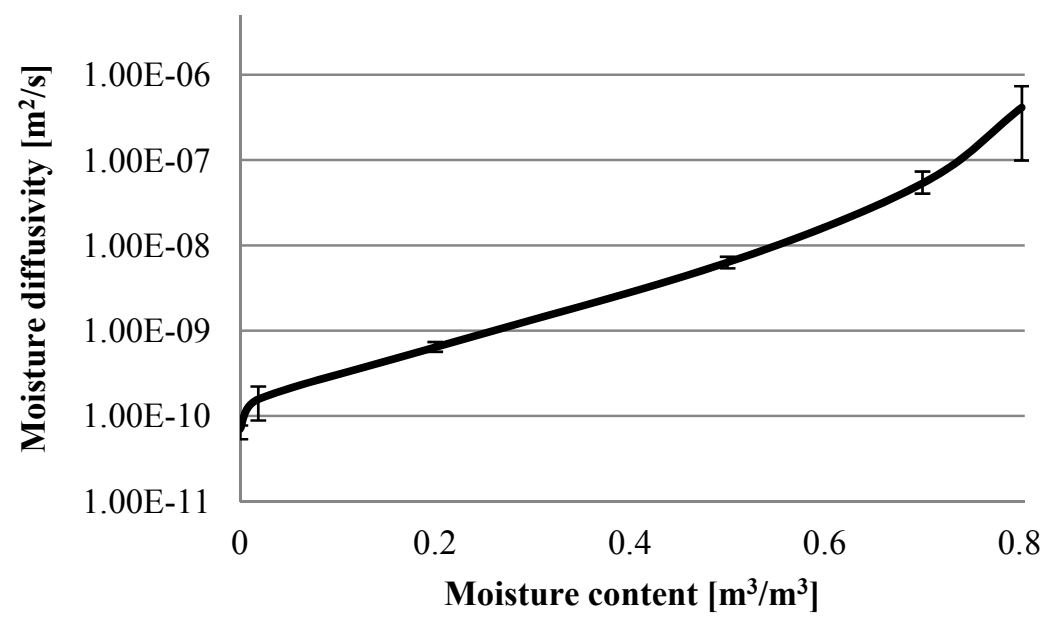

Figure 6: Moisture diffusivity of AAC (P4-500) at desorption.

In the future work it should be also very efficient to optimize the moisture diffusivity function. So far we used several isolated points for description of that function which may cause many inconveniences during optimization process. In the first place the optimization time is extended because of the size of the population. As mentioned above, the size of population in this paper was 70 chromosomes and 30 generations were created in total during optimization process. In each generation 70 numerical simulations were performed with average time consumption of 2 minutes and 33 seconds. This presents 2100 numerical simulations in total with optimization time of 321300 seconds (more than 3 days). If we find some mathematical approximation of the moisture diffusivity function that can be described using three or four parameters, the total time will rapidly compress. The other advantage of this approach is that the found function will be definitely smooth, which is not ensured for the approach using isolated points. From a mathematical point of view the moisture diffusivity function is not so easy to describe. At first glance it seems to have an exponential behavior, but in deeper look it is more complicated. In case we know the behavior of optimized function, one can use some of the existing description. But in case we do not know, some other and more flexible approach should be applied, that can be able to find mathematical description dynamically. The big 
potential may be hidden within genetic programming and therefore we should aim the future research at this field of science.

\section{Conclusions}

A combined computational-experimental approach for the determination of moisture diffusivity of AAC in drying phase was presented. In the experimental part the moisture content as a function of position and time was determined using a capacitance device. In the computational part, an innovative approach was used in the shape of genetic algorithms. Such approach allows finding moisture diffusivity not only for wetting phase, but drying phase as well. A practical applicability of the combined method was demonstrated on AAC typical for current low-energy building structures in Europe. Merits of this application are quite obvious: the determination of moisture diffusivity in dependence on moisture content at desorption presents a very substantial contribution to improvement of computer simulation of building performance. The computer simulations that cover the hysteretic effect of moisture transport and storage parameters are more reliable and may reveal the potential weak points of the construction that would be normally undetectable when neglecting the effect of hysteresis. It should be noted that in this paper we have also optimized the value of moisture transfer coefficient for the interior surface which belongs to very important parameters in hygrothermal modelling.

\section{Acknowledgement}

This research has been supported by the Czech Ministry of Education, Youth and Sports, under project No SGS12/104/OHK1/2T/11.

\section{References}

[1] Semerák, P., Černý, R. Kapacitní metoda měření vlhkosti stavebních materiálů. Stavební obzor, 6(4), pp. 102-103, 1997.

[2] Matano, C. On the Relation between the Diffusion Coefficient and Concentration of Solid Metals. Jap. J. Phys., 8, pp. 109-113, 1933.

[3] Drchalová, J., Černý, R. Non-Steady-State Methods for Determining the Moisture Diffusivity of Porous Materials. Int. Comm. in Heat and Mass Transfer, 25, pp. 109-116, 1998.

[4] Černý, R., Maděra, J., Kočí, J., Vejmelková, E. Heat and moisture transport in porous materials involving cyclic wetting and drying. Fourteenth International Conference on Computational Methods and Experimental Measurements, Algarve, Portugal. Southampton: WIT Press, pp. 3-12, 2009.

[5] Kelnar, J., Maděra, J., Černý, R. Computational Simulation of the Effect of Crystallization. Inhibitors on Salt Transport and Crystallization in Porous Materials. Computational Methods and Experimental Measurements XIII, Southampton: WIT Press, 46, pp. 367-375, 2007. 
[6] Jerman, M., Kočí, V., Výborný, J., Černý, R. Thermal and hygric properties of autoclaved aerated concrete. Thermophysics 2010. Brno: Brno University of Technology, pp. 102-108, 2010.

[7] Künzel, H.M. Simultaneous Heat and Moisture Transport in Building Components, PhD. Thesis. IRB Verlag, Stuttgart. 1995.

[8] Holland, J.H. Adaptation in natural and artificial systems. Internal report. Ann Arbor, University of Michigan, 1975.

[9] Goldberg, E.D. Genetic algorithms in search, optimization and machine learning. Reading, Addison-Wesley, 1989.

[10] Michalewicz, Z. Genetic algorithms + data structures = evolution programs, 3rd ed. Berlin, Springer, 1996.

[11] Kučerová, A. Identification of nonlinear mechanical model parameters based on soft computing methods. PhD thesis, Ecole Normale Supérieure de Cachan, 2007.

[12] Hrstka, O. (WWW), Homepage of SADE. http://klobouk.fsv.cvut.cz / ondra/sade/sade.htm 